\title{
Constraining Dark Halo Potentials with Tidal Tails
}

\section{Citation}

Dubinski, John, J. Christopher Mihos, and Lars Hernquist. 1999. “Constraining Dark Halo Potentials with Tidal Tails." The Astrophysical Journal 526 (2): 607-22. https:// doi.org/10.1086/308024.

\section{Permanent link}

http://nrs.harvard.edu/urn-3:HUL.InstRepos:41381816

\section{Terms of Use}

This article was downloaded from Harvard University's DASH repository, and is made available under the terms and conditions applicable to Other Posted Material, as set forth at http:// nrs.harvard.edu/urn-3:HUL.InstRepos:dash.current.terms-of-use\#LAA

\section{Share Your Story}

The Harvard community has made this article openly available.

Please share how this access benefits you. Submit a story.

\section{Accessibility}




\title{
Constraining Dark Halo Potentials with Tidal Tails
}

\author{
John Dubinski \\ CITA, University of Toronto, 60 St. George St., \\ Toronto, ON M5S 3H8, Canada \\ Lars Hernquist \\ Board of Studies in Astronomy and Astrophysics, UC Santa Cruz, \\ Santa Cruz, CA 95064 \\ J. C. Mihos \\ Department of Astronomy, Case Western Reserve University, \\ 10900 Euclid Ave, Cleveland, OH 44106
}

\begin{abstract}
Massive and extended dark halos can inhibit the formation of long tidal tails in galaxy collisions. We examine this effect using an extensive survey of simulations with different dark halo potentials to constrain halo properties of interacting galaxies. These constraints are compared to other observational limits and theoretical predictions of halo structure. The dark halos predicted by $\Omega=1$ cosmological models like CDM are too massive and extended to produce the long tidal tails seen in nearby galaxy collisions. There is also a conflict with the halo potentials inferred from satellite kinematics; such halos would likewise inhibit tail formation in galaxy collisions.
\end{abstract}

\section{Introduction}

When disk galaxies collide, the strong mutual tidal fields of their interaction momentarily transform the disks into open, bisymmetric spirals and catapult the outer disk stars in each galaxy onto long, arcing trajectories commonly called tidal tails and bridges (Toomre \& Toomre 1972; Wright 1972). Tidal bridges connect the merging pair and tidal tails continue to lengthen and thin out after a collision. Some tail stars may escape the system but most eventually fall back into the merger remnant. The currently interacting pairs in the Antennae (NGC 4038), the Mice (NGC 4676) and the merged NGC 7252 for example, all have tails which extend to distances $\sim 50-100 \mathrm{kpc}$ from the galaxies (Hibbard 1995). The Superantennae (IRAS 19254-7245) is an extreme case in which the tails span $350 \mathrm{kpc}$ from tip to tip (e.g., Mirabel, Lutz, \& Maza 1991).

A tidal tail can be thought of as a single trajectory shaped by the potential of the interacting pair since it arises from stars in the disk with similar orbital properties. Tidal tails can extend far out into the dark halo, perhaps out to $>200 \mathrm{kpc}$ in 3 dimensions. Tails were therefore recognized as a possible probe of the dark matter distribution in galaxies (Faber and Gallagher 1979) and 
their formation in simulations with dark halos has been the subject of much numerical work during the past decade (e.g. Negroponte \& White 1983; White 1982; Barnes 1988). The suggestion is that massive dark halos can inhibit the formation of tidal tails in two ways. First, the deeper potentials could lead to larger relative encounter velocities which are more impulsive and less resonant and therefore less effective at giving disk stars the velocity perturbation they need to be ejected as tails. Second, the deeper and steeper potential wells of more massive halos could trap tail stars by shortening their turn-around radius after ejection. Both effects would combine to make the maximum length of tidal tails smaller in the presence of a more massive dark halo. The observed maximum length of tails is therefore telling us something about the mass profile of the dark potential.

The length and kinematics of tidal tails depend on other factors besides the dark halo structure so simulation surveys are required to understand how they form in different circumstances. Other factors include the epoch of the collision, disk orientations, orbit orientation, orbital energy, galaxy mass ratio, and pericentric distance. The only way to study dark halo potential constraints is therefore to model the tail formation process as a function of dark halo parameters in galaxy collision N-body simulations (Dubinski, Mihos \& Hernquist 1996 [DMH96]). A complete parameter survey is out of reach so in our studies we have focussed on the ideal case of colliding equal mass, co-planar disks in a direct encounter. This is the geometry of the first galaxy collision ever simulated, namely that done by Holmberg in 1941 (before the computer age!). These encounters are strongly resonant and are the most effective at transferring energy and angular momentum to the tails and so the lengths of tails in these collisions probably represent an upper limit for all collision geometries. We have also examined more specific geometries which would produce facsimiles of the Antennae and NGC 7252 using different mass models (DMH96; Mihos, Dubinski, Hernquist 1998).

\section{Previous Results}

In previous work, we simulated galaxy collisions using four mass models labelled A, B, C and D with progressively more massive and more extended halos (DMH96). The models are self-consistent realizations containing an exponential disk, a truncated King model bulge and King model dark halo, constructed using the method of Kuijken \& Dubinski (1995). By construction, the disk and bulge mass distributions are held fixed and the inner rotation curve is kept nearly flat within 5 scale lengths in the model sequence. With this assumption, the mass ratio of dark matter to stars (disk + bulge) is 4:1, 8:1, 16:1 and 30:1 for models A, B, C, and D. Of course, more extended models with this mass ratio are also possible when dark halos have smaller circular velocities but we have only examined those with rotation curves which are approximately flat out to the halo truncation radius.

The results of this study are given in DMH96 and the main points are summarized here. Galaxy collisions with models A and B have no difficulty ejecting long tails $(l>100 \mathrm{kpc})$ while model $\mathrm{C}$ encounters eject tails of only moderate length $(l \sim 50-100 \mathrm{kpc})$ and model $\mathrm{D}$ collisions produce short tails 
$(l<50 \mathrm{kpc})$. When these simulations are carried through to the final merger to a state similar to NGC 7252, models A and B still have long tails while all of the stars in the tails in model $\mathrm{C}$ and $\mathrm{D}$ galaxies have fallen back into the merger remnant. On these grounds, we can rule out galaxies with mass distributions like models $\mathrm{C}$ and D as precursors of the Antennae, the Mice and NGC 7252. Collision geometries that produce facsimiles of the Antennae and NGC 7252 worked well with the low mass models A and B but failed with models C and D. Also, kinematic comparisons of NGC 7252 facsimiles to the real observations also rule out model $\mathrm{C}$ and $\mathrm{D}$ even when we include an "HI gas" disk (represented by test particles) extending out to 10 scale lengths (MDH98). Since models C and $\mathrm{D}$ had dark halo mass distributions very similar to those predicted from CDM or other $\Omega=1$ cosmological models, we argued that a lower $\Omega$ cosmology might be required to explain the observations. Indeed, small $\Omega$ cosmologies produce concentrated dark halos that look more like those in models A and B (Navarro et al. 1997).

\section{New Results}

How representative are these 4 models? They were constructed under the assumption that the flat inner rotation curve continues to large radii. Clearly, there are many other possibilities. For example, at large radii in an isothermal halo, $\phi \sim v_{c}^{2} \log r$ so $\partial \phi / \partial r \sim v_{c}^{2} / r$. If the disk contributes considerably more mass to the inner rotation curve the asymptotic value of $v_{c}$ may be smaller and so the outer potential will be shallower. Tidal tails may therefore be expelled to larger distances in this case since the potentials are not as effective a trap. This is just one example of how things can stray from our first 4 models.

The only way to understand the effects on tidal tail formation is to widen the survey to include other dark halo potentials. We have done just that by looking at 84 new models (Dubinski, Mihos, Hernquist [DMH98]). We have parameterized the dark halo using two models which have been motivated by cosmological simulations of dark halo formation: the Hernquist $(\mathrm{H})$ profile (Hernquist 1990; Dubinski \& Carlberg 1991) and the NFW profile (Navarro, Frenk \& White 1996). Both profiles have two free parameters, a mass (or effective mass within some radius in the case of the NFW profile since total mass formally diverges) and a scale length. The H-profile is:

$$
\rho(r)=\frac{M_{H} r_{H}}{2 \pi} \frac{1}{r\left(r+r_{H}\right)^{3}},
$$

where $M_{H}$ is the $\mathrm{H}$ mass and $r_{H}$ is the $\mathrm{H}$ scale length. The NFW profile is:

$$
\rho(r)=\frac{M_{s}}{4 \pi} \frac{1}{r\left(r+r_{s}\right)^{2}},
$$

where $M_{s}$ is the NFW effective mass (mass within $5.3 r_{s}$ ) and $r_{s}$ is the NFW scale length. Both profiles have peak circular velocities which we use to characterize them. For the $\mathrm{H}$ model $v_{H}^{2}=0.5 G M_{H} / r_{H}$ at $r=r_{H}$ and for the NFW model $v_{s}^{2}=0.46 G M_{s} / r_{s}$ at $r=2.16 r_{s}$. The velocity maxima of the two

dark halo rotation curves coincide when we use the mapping $r_{s}=0.46 r_{H}$ and 
$M_{s}=0.54 M_{H}$. The NFW model is more extended than the $\mathrm{H}$ model and has a steeper potential at larger radii, however, within the region where tails form the potentials are very similar. We found that there was very little difference in the results for both models so we only present the results of the NFW models here, although a more extensive comparison will be given elsewhere (DMH98).

In our previous work, we simulated the collisions with self-consistent treecode N-body simulations. The large number of models in this extended study precludes the use of self-consistent simulations at the moment so we use instead a restricted, 3-body method somewhat similar to Toomre \& Toomre's (1972) original scheme. We can get away with this since the tidal tails are essentially a kinematic phenomenon. The technique will be described elsewhere in detail (DMH98) but in brief it works as follows. The trajectories of the two interacting galaxies are calculated assuming an interaction potential with zero total energy which accounts for the extended mass distribution and is somewhat different than the parabolic orbits of point masses used by Toomre \& Toomre. The orbital decay of the galaxies is treated used Chandrasekhar's dynamical friction formula (Binney \& Tremaine 1987) using a value of the Coulomb logarithm, $\ln \Lambda=2$, found to fit the observed orbital decay well in a small number of self-consistent simulations. Finally, a test-particle realization of the orbital distribution of the disk is generated self-consistently according to the total galaxy potential for the models using the technique of Kuijken \& Dubinski (1995). The test particles are then integrated in the time-dependent gravitational field of the two rigid potentials moving along the pre-calculated collision trajectory. This technique produces tidal tail morphologies and kinematics remarkably similar to self-consistent simulations, so we apply it generally to models in our survey.

Halos are parameterized in our study by their scale length and circular velocity maximum. We have chosen six halo scale-lengths ranging from $r_{s}=1.2$ to 11.6 and seven circular velocities ranging from $v_{s}=0.5$ to 1.0 for a total of 42 models in the NFW study. We also looked at a similar set of $42 \mathrm{H}$ models. The disk exponential scale length and mass are both unity, so the disk's peak circular velocity is $v_{d}=0.62$ in these units. The bulge has a mass of $0.5 M_{d}$ and is the main contributor to the rotation curve within 1 scale length. This range of models broadly covers the inferred properties of dark halos around exponential galactic disks of Hubble type Sa-b similar to the Milky Way. Figure 11 shows the rotation curves within 10 scale-lengths.

Figure 2 presents the results of a series of planar, prograde galaxy collisions. Each collision is shown at the same time, $t=30$, after pericentric passage, corresponding to 5 disk orbital times at one scale length or 500 million years for the Milky Way. The size of each box is 80 scale lengths or about $300 \mathrm{kpc}$ for the Milky Way. The main results are that galaxies with low-mass, compact halos expel long tidal tails in collisions similar to those in observed interacting pairs while galaxies with high-mass extended halos expel very short tails unlike the Antennae or NGC 7525. The region where halos are similar to CDM predictions also produce relatively short tails in accord with our earlier conclusions. Galaxies with extended halos having smaller peak circular velocities (upper right corner) can also produce long tidal tails and bridges since the galaxies separate significantly after their encounter. The potential is shallow enough to allow long tails to fly off to large distances but the low central density of the halo does 


\section{NFW Halo Rotation Curves}

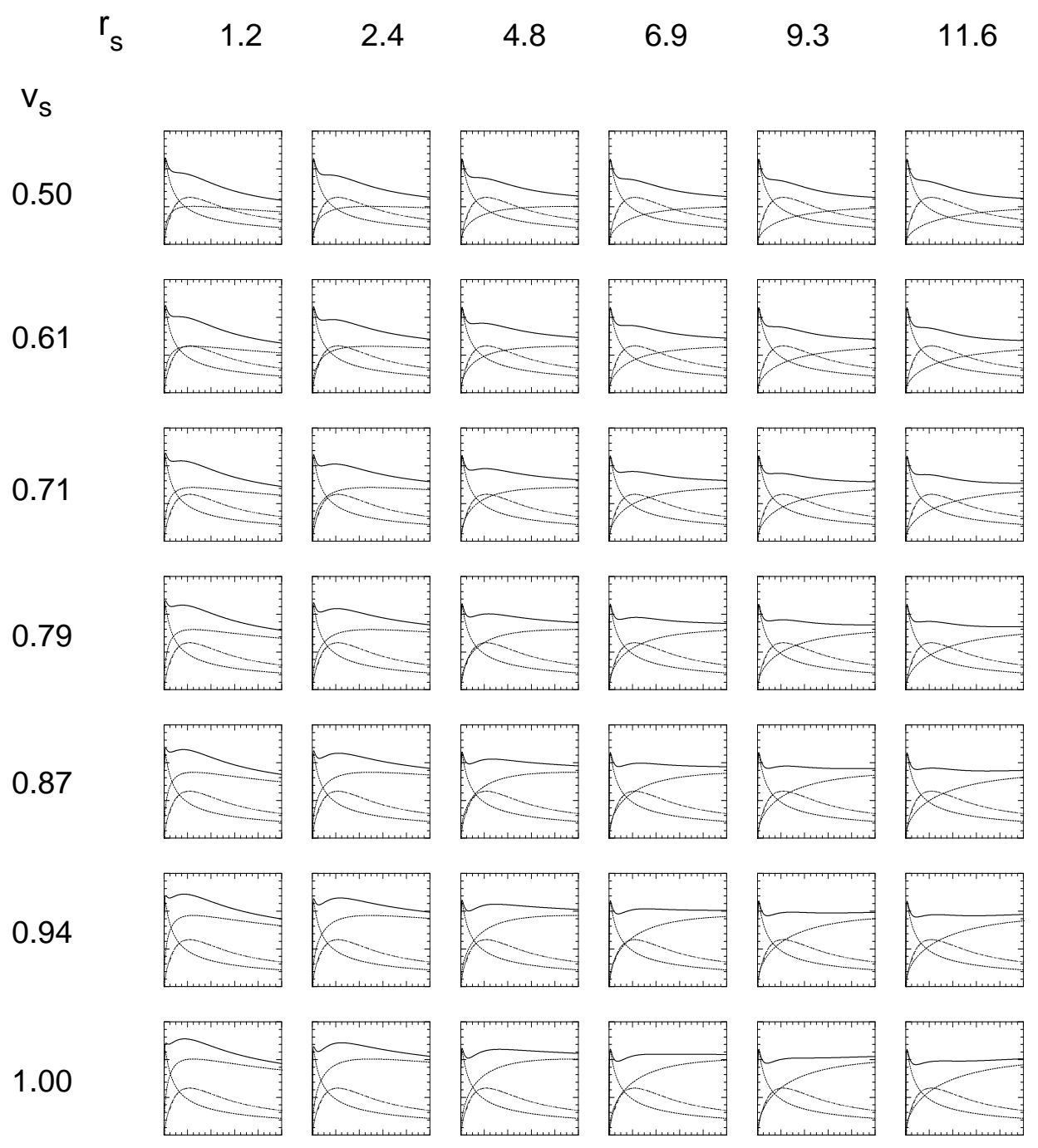

Figure 1. Rotation curves of the galaxy models in the collision survey. The contribution from the disk, bulge and halo as well as the net rotation curve are shown within 10 scale lengths for each model. A range of models is covered with compact low-mass halos (upper left corner) to high-mass, extended halos (lower right corner). 
Galaxy Collisions with NFW Halos

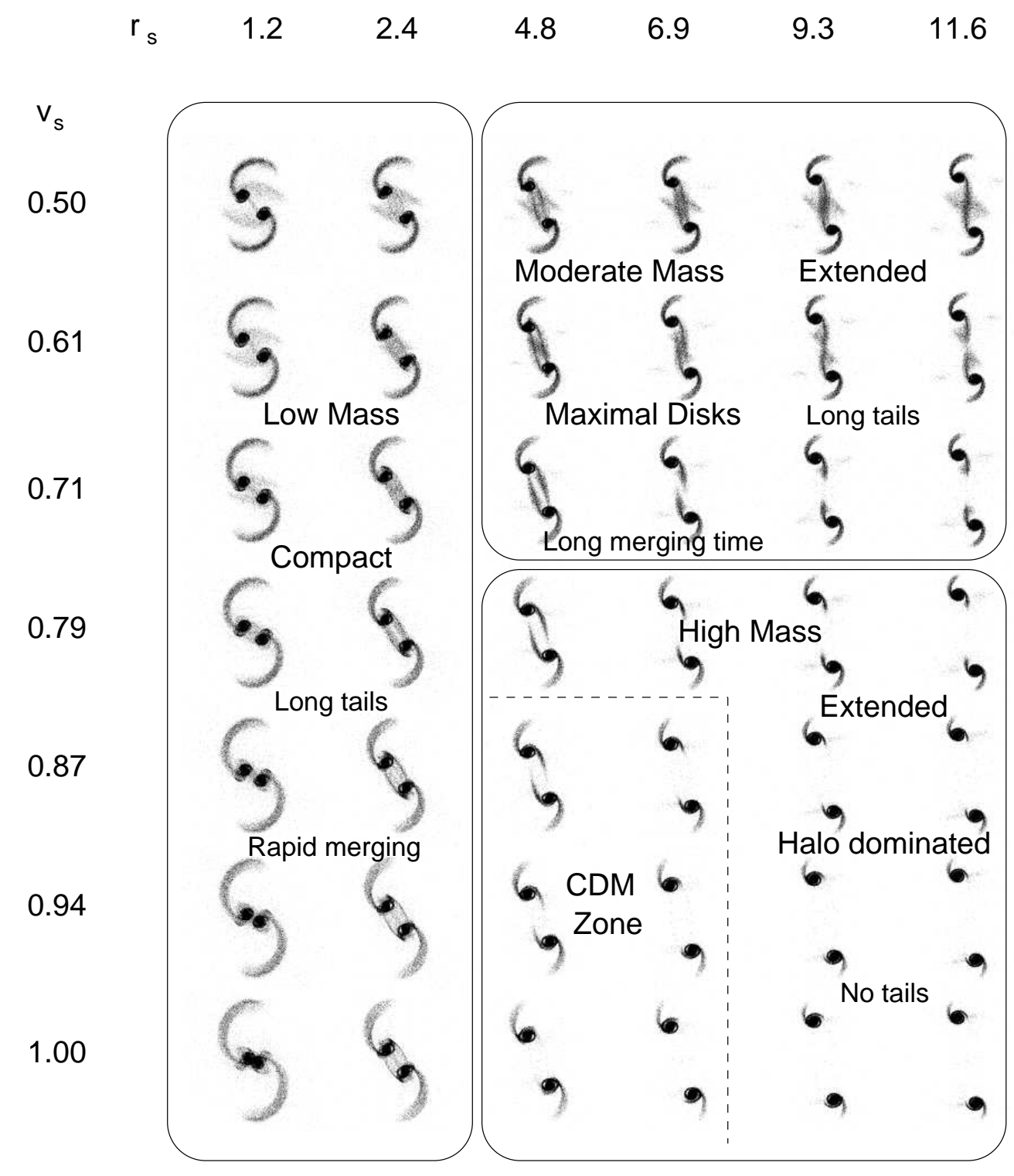

Figure 2. Tidal tails resulting from galaxy collisions in the model survey. See the text for a detailed description. 
not provide enough dynamical friction to slow down the galaxies significantly during their encounter in contrast to the models in the lower left corner where the central halo density is much higher. The interacting pair Arp 295 with its wide separation and long tails and connecting bridge has a similar morphology to galaxies in the upper right corner.

\section{Discussion and Conclusions}

This more extensive survey confirms our previous conclusion that collisions of galaxies with dark halos like CDM halos are ineffective at making long tidal tails. Halos which are compact and centrally concentrated with a range of circular velocities all make long tidal tails but extended halos can only make long tails if they have a small peak velocity i.e. a smaller contribution than the disk and bulge to the inner rotation curve. The successful galaxy models with extended halos are probably most similar to maximal and Bottema (1997) disk/halo models which are preferred by some rotation curve analysts. The main cosmological implication is that CDM and critical density universe models are not favored because their dark halos inhibit the formation of long tidal tails. More compact dark halos are predicted in low density universes, however, similar to models in the lower left of Figure 2 (Navarro et al. 1997).

The disagreement with the relatively large mass estimates in the Milky Way and external galaxies made through studies of satellite kinematics is harder to understand (e.g. Zaritsky et al. 1989; Kochanek 1996; Zaritsky \& White 1994; Zaritsky et al. 1997). Perhaps the difference is due to a selection effect galaxies chosen for satellite kinematics studies are well-isolated while interacting galaxies are obviously in pairs and not isolated. The different halos may reflect some cosmological variance resulting from differences in environment or initial conditions. In any case, the lower bounds of the confidence limits on halo masses from satellites overlap with range of galaxy models that make long tidal tails. Clearly, the systematic errors of these mass estimates should be studied in more detail, perhaps through comparison to cosmological simulations with adequate resolution to resolve satellites within their galactic halos. Galaxy interaction studies should also take their initial conditions from cosmological models instead

of the idealized models examined here. Perhaps a consistent picture will be found soon and the tails will stop wagging the dogs.

\section{References}

Barnes, J.E. 1988, ApJ, 331, 699

Binney, J., \& Tremaine, S. 1987, Galactic Dynamics (Princeton: Princeton University Press)

Bottema, R. 1997, A\&A, 328, 517

Dubinski, J. \& Carlberg, R.G. 1991, ApJ, 378, 496

Dubinski, J., Mihos, J.C., \& Hernquist, L. 1996, ApJ, 462, 576 (DMH96)

Dubinski, J., Mihos, J.C., \& Hernquist, L. 1998, in preparation (DMH98)

Faber, S.M., \& Gallagher, J.S. 1979, ARA\&A, 29, 409 
Hernquist, L. 1990, ApJ, 356, 359

Hibbard, J.E. 1995, Ph.D. thesis, Columbia University

Holmberg, E. 1941, ApJ, 94, 385

Kochanek, C. 1996, ApJ, 457, 228

Kuijken, K., \& Dubinski, J. 1995, MNRAS, 277, 1341

Mihos, J.C., Dubinski, J., \& Hernquist, L. 1998, ApJ, in press (MDH98)

Mirabel, I.F., Lutz, D., \& Maza, J. 1991, A\&A, 243, 367

Navarro, J.F., Frenk, C.S. \& White, S.D.M. 1996, ApJ, 462, 563

Navarro, J.F., Frenk, C.S. \& White, S.D.M. 1997, ApJ, in press

Negroponte, J., \& White, S.D.M. 1983, MNRAS, 205, 1009

Toomre, A., \& Toomre, J. 1972, ApJ, 178, 623

White, S. 1982, in The Morphology and Dynamics of Galaxies, ed. L. Martinet \& M. Mayor (Sauverny: Geneva Obs.), 289

Wright, A.E. 1972, MNRAS, 157, 309

Zaritsky, D., \& White, S.D.M. 1994, ApJ, 435, 599

Zaritsky, D., Smith, R., Frenk, C., \& White S.D.M. 1997, ApJ, 478, 39

Zaritsky, D., Olszewski, E.W., Schommer, R.A., Peterson, R.C., \& Aaronson, M. 1989, ApJ, 345, 759 